\title{
Appearance and Characterization of Lipoprotein X during Continuous Intralipid Infusions in the Neonate
}

\author{
E. Griffin, W. C. Breckenridge, A. Kuksis, M. H. Bryan, and A. ANGel, \\ Research Institute, The Hospital for Sick Children, Lipid Research Clinic, \\ Department of Medicine, University of Toronto, Banting and Best, Department \\ of Medical Research, University of Toronto, and Department of Medicine, \\ University of Toronto, Toronto, Canada
}

A B S T RACT The development of hyperphospholipidemia and hypercholesterolemia was studied in infants that required total parenteral nutrition and given a continuous infusion of Intralipid, $(1-4 \mathrm{~g} / \mathrm{kg}$ body wt per $24 \mathrm{~h}$. Detailed studies were carried out on infusion periods lasting 1-10 d. After $24 \mathrm{~h}$ there was a marked increase in plasma free cholesterol $(68 \%)$ and phospholipid $(\mathbf{7 7 \%})$ concentrations. Based on the amount of cholesterol in Intralipid, and the rate of infusion, it was estimated that at least $50 \%$ of the plasma cholesterol increment during 64-h infusions was derived from endogenous sources. By contrast, the hyperphospholipidemia could be attributed to the Intralipid as the rise in plasma was calculated to be equivalent to only $16 \%$ of the exogenous phospholipid infused. Approximately $10 \%$ of the phospholipid in Intralipid was in a triglyceride-free mesophase form with a free cholesterol:phospholipid molar ratio of 0.063 . There were no systematic changes in plasma concentrations of cholesterol ester or triglyceride during Intralipid infusions. The increase in free cholesterol and phospholipid was localized in the low density lipoproteins $(d=1.006-1.063 \mathrm{~g} / \mathrm{ml})$. The presence of lipoprotein $\mathrm{X}$ $(\mathrm{Lp}-\mathrm{X})$ in the low density lipoprotein fraction was demonstrated by electrophoresis in agar and by isolation and chemical characterization with hydroxylapatite chromatography. Isoelectric focusing of ureasoluble protein of $\mathrm{Lp}-\mathrm{X}$ revealed that albumin and apolipoproteins CII and CIII were major components, whereas apolipoprotein $\mathrm{E}$ and $\mathrm{AI}$ were minor constituents. The abnormal lipoprotein was apparent by $16 \mathrm{~h}$

Dr. Griffin was a Fellow of the Medical Research Council of Canada.

Address reprint request to Dr. Angel, University of Toronto, Toronto, Ontario, Canada, M5S 1 A8.

Received for publication 10 July 1978 and in revised form 27 July 1979. during $64 \mathrm{~h}$ of infusion. After $6 \mathrm{~d}$ of continuous infusions the free cholesterol in $\mathrm{Lp}-\mathrm{X}$ was $30 \pm 10 \mathrm{mg} / \mathrm{dl}$ $($ mean $\pm \mathrm{SD}$ ), which represents a total Lp-X mass of $90 \mathrm{mg} / \mathrm{dl}$. After cessation of the infusion, Lp-X, as monitored by electrophoresis in agar, disappeared within 72-96 h. Thus, during infusion of Intralipid in infants at rates commonly employed, the capacity of the clearance mechanisms for phospholipid are exceeded, which causes the accumulation of phospholipid and free cholesterol in the form of Lp-X particles. It is suggested that mesophase phospholipids in Intralipid may play a significant role in this process.

\section{INTRODUCTION}

Although fat emulsions are needed to supply additional calories and to avoid essential fatty acid deficiency during intravenous nutrition in the neonate (1), their use has been associated with various hyperlipidemias. Intralipid, the most commonly used parenteral fat solution, is an emulsion of soybean triglyceride that contains $1.2 \%$ egg lecithin as a stabilizing agent. Hypertriglyceridemia, defined as plasma triglyceride concentration exceeding $100 \mathrm{mg} / \mathrm{dl}$, has been noted in small-for-gestational-age infants $(2,3)$, and in the premature, $<32$ wk $(3,4)$; and is manifested by a delay in clearing bolus injections or infusions $(4 \mathrm{~h})$ of Intralipid. This apparent intolerance to parenteral lipid has been attributed to reduced hydrolysis of Intralipid triglyceride as well as to a diminished uptake and utilization of the liberated FFA in peripheral tissue. In recent reports a rise in plasma free cholesterol and phospholipid concentrations has been observed in both the neonate $(5,6)$ and adults $(7-9)$ after infusions of Intralipid. In view of these observations a detailed study was undertaken to investigate the alterations in plasma lipids and lipoproteins during continuous administration of Intralipid to neonates dependent 
upon total parenteral nutrition (TPN). ${ }^{1}$ On the basis of this study it is suggested that the hypercholesterolemia is a consequence of phospholipid overload, which causes the accumulation of a lipoprotein with chemical composition and electrophoretic properties of lipoprotein $\mathrm{X}$.

\section{METHODS}

Patients. The infants studied required TPN for a variety of congenital and acquired gastrointestinal anomalies. All were appropriate for gestational age that ranged from 26- to 40-wk gestation at birth, and were from 4-d- to 3-wk-old when Intralipid (Cutter Medical, Div. Cutter Laboratories, Berkeley, Calif.) was started. Intralipid ${ }^{2}$ (containing per $100 \mathrm{ml}: 10 \mathrm{~g}$ soybean triglyceride, $1.2 \mathrm{~g}$ egg lecithin, and $2.5 \mathrm{~g}$ glycerol) was administered via a scalp vein needle using a Sage syringe pump (model 255, Sage Instruments Div., Orion Research Inc., Cambridge, Mass.) to maintain a constant rate of infusion. The infants received glucose and casein hydrolysate for at least $24 \mathrm{~h}$ before and during the period of Intralipid infusion. None of the infants was acidotic, septic, or had bilirubin concentrations exceeding $5 \mathrm{mg} / 100 \mathrm{ml}$. Infants with minor elevations in direct reacting bilirubin $(<2 \mathrm{mg} / 100 \mathrm{ml})$ were included because these infants did not differ from those in whom there was no such increase.

Based on Intralipid tolerance tests, individual infusion rates were calculated ${ }^{3}$ for the majority of infants studied as follows: a bolus injection of $200 \mathrm{mg}$ of Intralipid triglyceride/ $\mathrm{kg}$ body wt was given; the fractional elimination constant, $K_{2}$, and the Intralipid volume of distribution were determined, and these values were applied to the formula of the plateau principle $(10,11)$ to calculate an infusion rate to achieve a steady plasma Intralipid concentration of $100 \mathrm{mg} / \mathrm{dl}$ or less. This target concentration was chosen to avoid hyperlipidemia (12). ${ }^{3}$ All the infants in this study received, by continuous infusion, 1-4 g Intralipid triglyceride/ $\mathrm{kg}$ body wt per $\mathrm{d}$.

Lipoprotein analyses. Heel prick blood samples $(200 \mu \mathrm{l})$ were taken into capillary tubes that contained EDTA or heparin for studies involving agarose electrophoresis or lipid analyses, respectively. For lipoprotein isolation 1.3-2.0 $\mathrm{ml}$ of blood was collected into EDTA by venous puncture. Plasma lipoprotein fractions were isolated (13) in a Beckman rotor (40.3, Beckman Instruments, Inc., Spinco Div., Palo Alto, Calif.) fitted with a Teflon (E. I. DuPont, de Nemours \& Co., Inc., Wilmington, Del.) insert that would accept $2.0-\mathrm{ml}$

\footnotetext{
${ }^{1}$ Abbreviations used in this paper: HDL, high density lipoprotein(s); LCAT, lecithin-cholesterol acyltransferase; LDL, low density lipoprotein(s); Lp-X, lipoprotein $\mathrm{X}$; TMU, tetramethylurea; TPN, total parenteral nutrition; VLDL, very low density lipoprotein(s).

${ }^{2}$ Detailed analyses of Intralipid (three preparations) revealed the presence of free cholesterol $(4 \mathrm{mg} / \mathrm{g}$ Intralipid triglyceride) as well as plant sterols $(2 \mathrm{mg} / \mathrm{g}$ Intralipid triglyceride). Two Intralipid preparations were subjected to ultracentrifugation at saline density $\left(1.6 \times 10^{6} \mathrm{~g} \mathrm{~min}\right)$. Analysis of the supernatant and infranatant fractions obtained after tube slicing revealed that $8-12 \%$ of the total phospholipid and free cholesterol was associated with the infranatant fraction as a triglyceride-free micelle. The molar ratio of free cholesterol:phospholipid in the triglyceride-rich supernatant and the infranatant mesophase was 0.063 .

${ }^{3}$ Griffin, E. A., M. H. Bryan, and A. Angel. Intralipid tolerance tests and continuous infusion in neonates. Manuscript submitted for publication.
}

cellulose nitrate centrifuge tubes. Plasma $(0.6-1.0 \mathrm{ml})$ was overlayered with saline, $\mathrm{pH} 7.4(d=1.006 \mathrm{~g} / \mathrm{ml})$ and centrifuged at $106,000 \mathrm{~g}$ for $18 \mathrm{~h}$. A supernatant fraction $(0.8 \mathrm{ml})$ and an infranatant fraction $(1.2 \mathrm{ml})$ were quantitatively collected by tube slicing. The infranatant fraction was adjusted to $d 1.063$ and centrifuged at $106,000 \mathrm{~g}$ for $24 \mathrm{~h}$ to obtain low density lipoproteins (LDL) in the supernatant fraction. For studies involving protein analyses, LDL was washed once. The infranatant was adjusted to $d=1.21 \mathrm{~g} / \mathrm{ml}$ and centrifuged for $42 \mathrm{~h}$ at $106,000 \mathrm{~g}$ to obtain high density lipoproteins (HDL).

The lipoproteins of $d$ 1.006-1.063 were further resolved by hydroxylapatite column chromatography as described by Kostner et al. (14) for the isolation of lipoprotein X (Lp-X) from cholestatic patients. In these studies, columns were eluted at $0.1 \mathrm{M}$ phosphate buffer ( $\mathrm{pH} 6.8$ ) to obtain $\mathrm{Lp}-\mathrm{X}$ and at $0.65 \mathrm{M}$ phosphate ( $\mathrm{pH} 6.8$ ) to obtain "residual LDL." Because initial studies demonstrated that the elution profiles monitored by optical density $(280 \mathrm{~nm})$ or cholesterol content were identical, optical density was routinely used.

Lipoprotein electrophoresis on agarose gel was carried out by the method of Maguire and Breckenridge (15), whereas electrophoresis on agar, for the detection of $\mathrm{Lp}-\mathrm{X}$, was performed as described by Seidel et al. (16). Apoproteins from various lipoproteins were analyzed by polyacrylamide gel electrophoresis after treatment of the lipoprotein with tetramethylurea (TMU) as described by Kane (17). Apoprotein that was insoluble in TMU was assumed to be apoprotein (apo)B. Apoprotein components were identified on polyacrylamide gels by comigration with purified apoproteins or by their relative electrophoretic mobility.

Apoproteins were also characterized by isoelectric focusing. Lyophilized lipoproteins were delipidated with three extractions of ethanol:ether $3: 1(\mathrm{vol} / \mathrm{vol})$. The dried residue was taken up in Tris- $\mathrm{HCl}$ buffer $(1 \mathrm{mM}, \mathrm{pH})$ that contained $8 \mathrm{M}$ urea and $1 \mathrm{mg}$ dithiothreitol/100 $\mu \mathrm{g}$ protein. Isoelectric focusing was completed essentially according to the method of Pagnan et al. (18) with the following modifications. A linear gradient between pH 3 and 7 was achieved by using ampho-

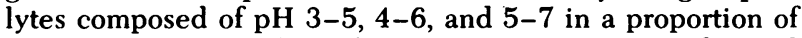
1:2:2 (vol/vol per vol). Gels $(0.5 \times 12 \mathrm{~cm})$ were prefocused for $1 \mathrm{~h}$ at $100 \mathrm{~V}$. The samples $(50-$ to $100-\mu \mathrm{g}$ urea-soluble protein) were applied in $8 \mathrm{M}$ urea and overlayered with an aqueous solution that contained $1 \%$ ampholyte mixture and $5 \%$ sucrose. The gels were focused for $250 \mathrm{~V}$ for $16 \mathrm{~h}$ and then stained as described elsewhere (19). For determination of the $\mathrm{pH}$ gradient a gel, lacking sample was subjected to focusing and then cut into 5 - $\mathrm{mm}$ segments that were placed in distilled water $(0.5 \mathrm{ml})$. The $\mathrm{pH}$ was recorded after $1 \mathrm{~h}$. Apoproteins were identified by comparison to published values for their $\mathrm{pI}$ and by isoelectric focusing with purified apoproteins.

The various lipoprotein fractions were also assessed by double diffusion against anti-human albumin (Miles Laboratories Inc., Elkhart, Ind.) and anti-human LDL. The latter was prepared in rabbits by injection of human LDL $(d=1.030-$ $1.050 \mathrm{~g} / \mathrm{ml}$ ) in an equal mixture with Freund's adjuvant.

Protein was estimated by the method of Lowry et al. (20) with bovine serum albumin as a standard. Lipoprotein fractions and standards were extracted with diethyl ether after color development. The protein content in TMU extracts was determined as described by Kane (17) except that TMU was used in the standards.

Lipid analysis by gas-liquid chromatography. Plasma or lipoprotein fractions were assayed for triglycerides, esterified cholesterol, free cholesterol, and phospholipids by an automated gas chromatographic procedure as previously described (21). For this purpose the plasma $(50 \mu \mathrm{l})$ was digested under diethyl ether with phospholipase $\mathbf{C}$ (Clostrid- 
ium welchii), which converted the lecithins and sphingomyelins into the corresponding diglycerides and ceramides, and any lysolecithins into the monoglycerides. After extraction with chloroform-methanol, the mixed neutral lipids were treated with trimethylchlorosilane and hexamethyldisilazane, which converted any FFA into the trimethylsilyl esters, the free cholesterol, diglycerides, and ceramides into the corresponding trimethylsilyl ethers, and any monoglycerides into the ditrimethylsilyl ethers. The neutral lipid mixture was separated and quantitated in the presence of tridecanoin as internal standard by automated high-temperature gasliquid chromatography with $20 \times 1 / 16$-in i.d. stainless steel tubes that contained 3\% OV-1 (a methyl siloxane polymer) on Gas-Chrom Q (100-200 mesh) (Applied Science Laboratories Inc., State College, Pa.). The gas chromatograph was the Hewlett-Packard automated gas chromatographic system 5700 A (Hewlett-Packard Co., Palo Alto, Calif.), which included an automatic sampler, low mass oven with temperature programmer, electronic integrator, and a dual flame-ionization detector. The integrator output was simultaneously recorded on a strip chart recorder and a punched paper tape that encoded the peak retention times and areas in computer-compatible code. The tape was subsequently used for off-line data processing by means of computer programs (21). Appropriate calibration and conversion factors were used for differences in recovery, flame-ionization response, and the chemical form of analysis of the different plasma lipids. The precision of the methodology was $3-5 \%$ for the estimate of lipid classes, whereas the limit of detection of lipid classes was $\sim 10 \mu \mathrm{g} / \mathrm{dl}$.

A paired $t$ test was used to compare lipid results in plasma before, during, and after Intralipid administration.

\section{RESULTS}

Initial observations (Fig. 1) in neonates receiving continuous Intralipid infusions revealed a marked rise in plasma free cholesterol that correlated significantly

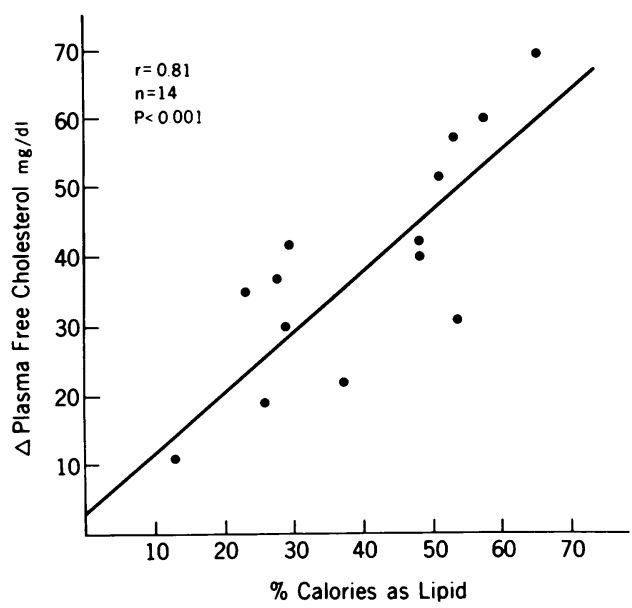

FIGURE 1 Effect of Intralipid load on plasma free cholesterol concentration in neonates $(n=14)$. All the infants received continuous Intralipid infusions $(1-8 \mathrm{~g} / \mathrm{kg}$ body $\mathrm{wt}$ ) over a period of $20-24 \mathrm{~h}$. The total nonfat calories were constant for any given subject and ranged from 28 to $76 \mathrm{kcal} / \mathrm{kg}$ body wt. The increase in plasma free cholesterol was linearly related $(P<0.001)$ to the amount of Intralipid given on a daily basis. with the amount of Intralipid infused $(P<0.001)$ over the time period. These observations prompted more detailed time-related studies to assess the effect of Intralipid on plasma lipids and lipoproteins in infants requiring TPN.

Plasma lipids. In Fig. 2 the changes in total lipid profiles of an infant are shown before Intralipid infusion (panel A); after $24 \mathrm{~h}$ (panel B) and after $2.5 \mathrm{mo}$ (panel C) on Intralipid infusion; as well as 3 mo after discontinuation of Intralipid (panel D). Before infusion the major plasma lipid constituents were free cholesterol (C27), phospholipids (C34-C40), and cholesterol esters (C43 and C45). After $24 \mathrm{~h}$ of Intralipid infusion there was a marked increase in free cholesterol and phospholipid concentration but no increase in triglycerides (C50-C54). By 2.5 mo plant sterols became evident as the amounts of campesterol (C28) and $\beta$-sitosterol (C29) were more obvious than at $24 \mathrm{~h}$ of infusion. In this individual there was a marked decrease in cholesterol ester, but this was not a systematic change because increases in cholesterol esters were observed in several short-term studies $(7 \mathrm{~d})$. It should be noted parenthetically that the Intralipid used in this study contained small amounts of plant sterols $(2 \mathrm{mg} / \mathrm{g}$ Intralipid triglyceride) as well as cholesterol $(4 \mathrm{mg} / \mathrm{g}$ Intralipid triglyceride). 3 mo after discontinuation of the Intralipid the proportions of free cholesterol, phospholipid, and cholesterol ester had returned to preinfusion levels.

A more extensive study of plasma lipid composition before and at 20-24 h of continuous Intralipid infusion was undertaken in 10 infants (Table I). A significant increase $(P<0.01)$ occurred in plasma free cholesterol and phospholipid concentrations, but little change in concentration was evident for esterified cholesterol and triglycerides. The plasma lipid values of the infants before Intralipid infusion were indistinguishable from a group of nine normal infants on formula feeds. The appearance of small amounts of plant sterols (campesterol and $\beta$-sitosterol) were observed in 5 of the 10 infants infused over 20-24 h.

To identify the early phases of free cholesterol and phospholipid accumulation, the plasma phospholipid and free cholesterol concentrations were followed (Fig. 3) over a 64 -h period in a group of five infants receiving continuous Intralipid infusions. A rise in plasma phospholipid and free cholesterol was seen at very early times $(4 \mathrm{~h})$ after starting the infusion. It was apparent that during the initial $16 \mathrm{~h}$ the rise in phospholipid was greater than the rise in free cholesterol - but thereafter they rose in parallel. The increase in plasma free cholesterol concentration could not be attributed solely to the cholesterol in Intralipid, which contained $4 \mathrm{mg}$ cholesterol/g triglyceride. A calculation (Fig. 3) of the increment in free cholesterol potentially attributable to the infused Intralipid was 


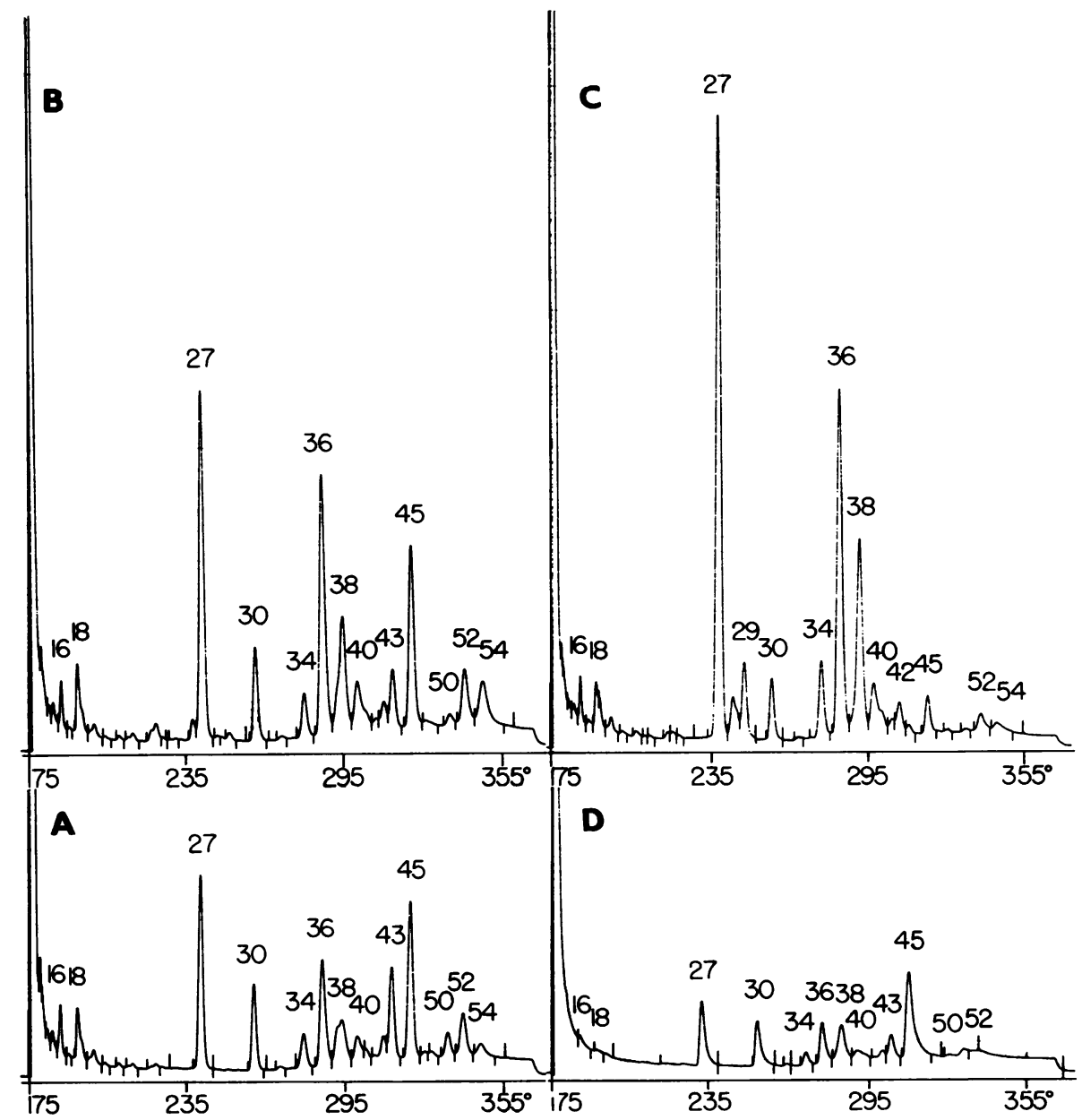

FigUre 2 Total plasma lipid profiles of an infant. (Panel A) before Intralipid infusion; (Panel B) after $24 \mathrm{~h}$ and (Panel C) after 2.5 mo of Intralipid administration (2 $\mathrm{g}$ Intralipid triglyceride/kg body wt per $24 \mathrm{~h}$ ); (panel D) 3 mo after discontinuation of TPN. Samples, $1 \mu$ l of approximately a $1 \%$ solution of neutral lipids in the silylation mixture; attenuation, $1 / 100$ full sensitivity; carrier gas, nitrogen at $80 \mathrm{ml} / \mathrm{min}$; detector, $350^{\circ} \mathrm{C}$; column temperature programmed at $6^{\circ} \mathrm{C} / \mathrm{min}$. Other conditions are given in text. The numbering system refers to the number of carbons in each lipid moiety represented by each separate peak. Peak 30, tridecanoin is the internal standard. Peaks 16 and 18 are trimethylsilyl esters of $C_{16}$ and $C_{18}$ fatty acids. Peaks 27, 28 (unmarked between 27 and 29 in panel C), and 29 (panel C) are trimethylsilyl ethers of cholesterol, campesterol, and $\beta$-sitosterol, respectively. Peaks 30-40 are acyl carbons. Peaks 43-45 are cholesterol esters of $C_{16}-C_{18}$ fatty acids. Peaks 50-54 are triglycerides containing 50-54 acyl carbons per glyceride molecule. A sharp increase in free cholesterol (peak 27) and phospholipids (peaks 36-40) in panels B and C during Intralipid infusion and its normalization 3 mo later in panel $D$ is evident.

made assuming that all Intralipid cholesterol was distributed in the plasma and that the plasma volume was $50 \mathrm{ml} / \mathrm{kg}$ body wt. No more than $40 \%$ of the increment could have come from the exogenous source. Similar calculations were made for the increment in plasma phospholipids. The increment after $64 \mathrm{~h}$ represents only $16 \%$ of that potentially available from the total phospholipid administered via Intralipid. The changes in cholesterol ester were variable and showed no specific trend (Fig. 3).
Alterations in lipoprotein composition. The increments in plasma free cholesterol and phospholipid were confined almost entirely to an increase (sixfold) in the concentration of LDL $(d=1.006-1.063 \mathrm{~g} / \mathrm{ml}$ as shown in Fig. 4). No significant changes occurred in absolute amounts of free cholesterol and phospholipids in HDL and very low density lipoproteins (VLDL). After cessation of Intralipid ( $5 \mathrm{wk}$ ) the lipoprotein composition and concentrations had returned to preinfusion values with the exception of HDL. The average 
TABLE I

Effect of Continuous Intralipid Infusion on Plasma Lipid Concentrations in Infants*

\begin{tabular}{|c|c|c|c|c|c|c|}
\hline \multirow[b]{2}{*}{ Experiment $t$} & & \multicolumn{5}{|c|}{ Component } \\
\hline & & $\begin{array}{c}\text { Free } \\
\text { cholesterol }\end{array}$ & $\begin{array}{l}\text { Esterified } \\
\text { cholesterol }\end{array}$ & $\begin{array}{c}\text { Plant } \\
\text { sterols } \S\end{array}$ & Phospholipid & Triglyceride \\
\hline & $n$ & & & $\mathrm{mg} / 100 \mathrm{ml}$ & & \\
\hline A (a) Pre-Intralipid infusion & 10 & $56.9 \pm 8.06$ & $87.5 \pm 9.4$ & 0 & $167 \pm 20$ & $136 \pm 19$ \\
\hline (b) 20-24 h of Intralipid infusion & 10 & $95.5 \pm 8.2$ & $82.8 \pm 5.3$ & $3.82 \pm 1.6$ & $295.2 \pm 21$ & $171 \pm 30$ \\
\hline B Formula fed (control) & 9 & $45.4 \pm 4.9$ & $89.2 \pm 17.2$ & 0 & $161.1 \pm 14.4$ & $127 \pm 18$ \\
\hline
\end{tabular}

* Total lipid profiles analyzed by gas-liquid chromatography as in legend of Fig. 2.

$\$$ (A) The 10 infants who were studied $(a)$ before and $(b)$ at $20-24 \mathrm{~h}$ of continuous Intralipid infusion of $2-4 \mathrm{~g} / \mathrm{kg}$ per $\mathrm{d}$ had a gestational age of $34.5 \pm 1.2 \mathrm{wk}($ mean $\pm \mathrm{SEM})$ and were $8 \pm 2 \mathrm{~d}$ old $(\mathrm{mean} \pm \mathrm{SEM})$. Five of the infants had received no feeds whereas the other five who had previously been commenced on formula feeds were fasting for at least $24 \mathrm{~h}$ before the study. (B) Nine fed infants receiving 5-10 $\mathrm{g}$ fat $/ \mathrm{kg}$ per $\mathrm{d}$ from formula had a gestational age of $33.4 \pm 1.5 \mathrm{wk}$ (mean $\pm \mathrm{SEM}$ ) and were aged $11 \pm 2 \mathrm{~d}(\mathrm{mean} \pm \mathrm{SEM})$. Significant differences $P<0.01$ were obtained with paired $t$ test for lipid values $A(a)$ and $(b)$. No significant differences were obtained between (B) formula-fed infants and (A) (a) pre-Intralipid infusion infants.

$\S$ Plant sterols (campesterol and $\beta$-sitosterol) appeared in 5 of the 10 infants and the mean of the 5 is shown.

content of cholesterol ester in this lipoprotein was considerably higher than the amount observed before or during Intralipid infusion but there was extensive intraindividual variation. However, the ratio of free cholesterol:esterified cholesterol dropped from 3.48 \pm 0.51 during the infusion to $0.45 \pm 0.06$ (mean $\pm S E M$ ) after the infusion. Before infusion this ratio was 4.4 \pm 1.38 . It is noteworthy that the lipoprotein had an abnormally high free cholesterol esterified cholesterol ratio even before infusion.

The effect of Intralipid infusion on lipoprotein composition was further examined by agarose gel electrophoresis. In preinfusion plasma (Fig. 5A) $\beta$-lipoproteins (LDL), pre- $\beta$ - (VLDL), and $\alpha$ - (HDL) bands were present and the origin was clear which indicates an absence of chylomicrons. After $64 \mathrm{~h}$ of Intralipid infusion (Fig. 5B) lipid-staining material was noted at the origin which indicates the presence of Intralipid. There was also a marked increase in the intensity of the $\beta$-band as well as a new, slow-migrating band just behind LDL. The appearance of the slow migrating band together with the increased concentrations of free cholesterol and phospholipid in the LDL fraction suggested the possible presence of Lp-X, which has been identified in the plasma of cholestatic patients (22). This suggestion was substantiated by agar electrophoresis where Lp-X migration is cathodal (Fig. 6). Fig. 6 also shows the appearance of $\mathrm{Lp}-\mathrm{X}$ on agar electrophoresis coinciding with the accumulation of free cholesterol and phospholipid in plasma during a 64-h infusion of Intralipid. Lp-X starts to appear at $16 \mathrm{~h}$ along with the parallel increases in free cholesterol and phospholipid (Fig. 3), and accumulates progressively during the remainder of the study period as judged by the intensity of the Lp-X band. The

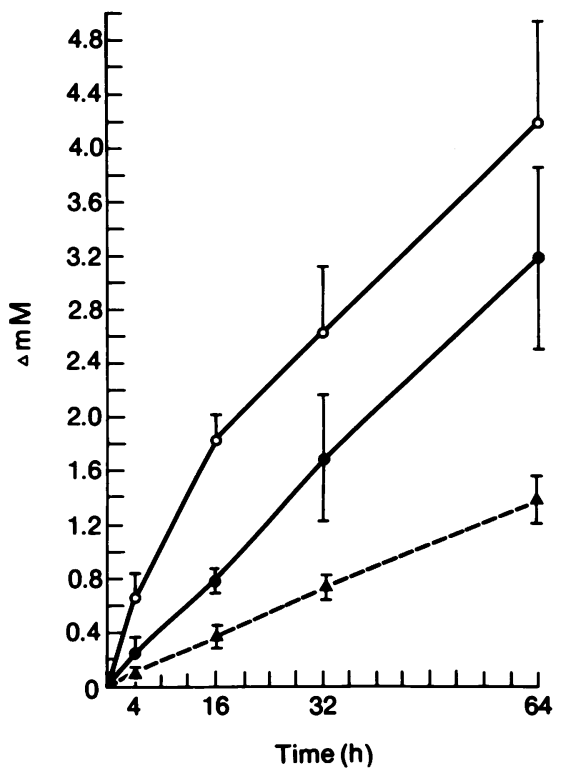

Figure 3 Changes in plasma free cholesterol and phospholipid during continuous intralipid infusions. Five neonates were given 1-4 g Intralipid triglyceride/kg body wt per $24 \mathrm{~h}$. Results are shown as means and standard error of the mean. (O) Plasma phospholipids; (O) plasma free cholesterol; (வ) estimate of plasma cholesterol increment potentially available from cholesterol content of Intralipid (4 mg cholesterol/g triglyceride) and distributing only in the plasma compartment (plasma volume assumed to be $50 \mathrm{ml} / \mathrm{kg}$ body wt). The increment in phospholipid due to Intralipid phospholipid under the same assumption was not included because of the much greater scale. The change in plasma phospholipid in millimolars, if all Intralipid phospholipid had remained in plasma, would be: $4 \mathrm{~h}, 1.5 ; 16 \mathrm{~h}, 6.0 ; 32 \mathrm{~h}, 12.0$; and $64 \mathrm{~h}, 24.0$. Changes in cholesterol ester, which were variable and not significant, were as follows: (mean and range in parenthesis, $\mathrm{mg} / 100 \mathrm{ml}), 4 \mathrm{~h},-5(-2$ to -5$) ; 16 \mathrm{~h},+1.3(-14$ to +16$) ; 32 \mathrm{~h}$, $+6.2(-5$ to +16$)$; and $64 \mathrm{~h},+14.4(-18$ to +61$)$. 


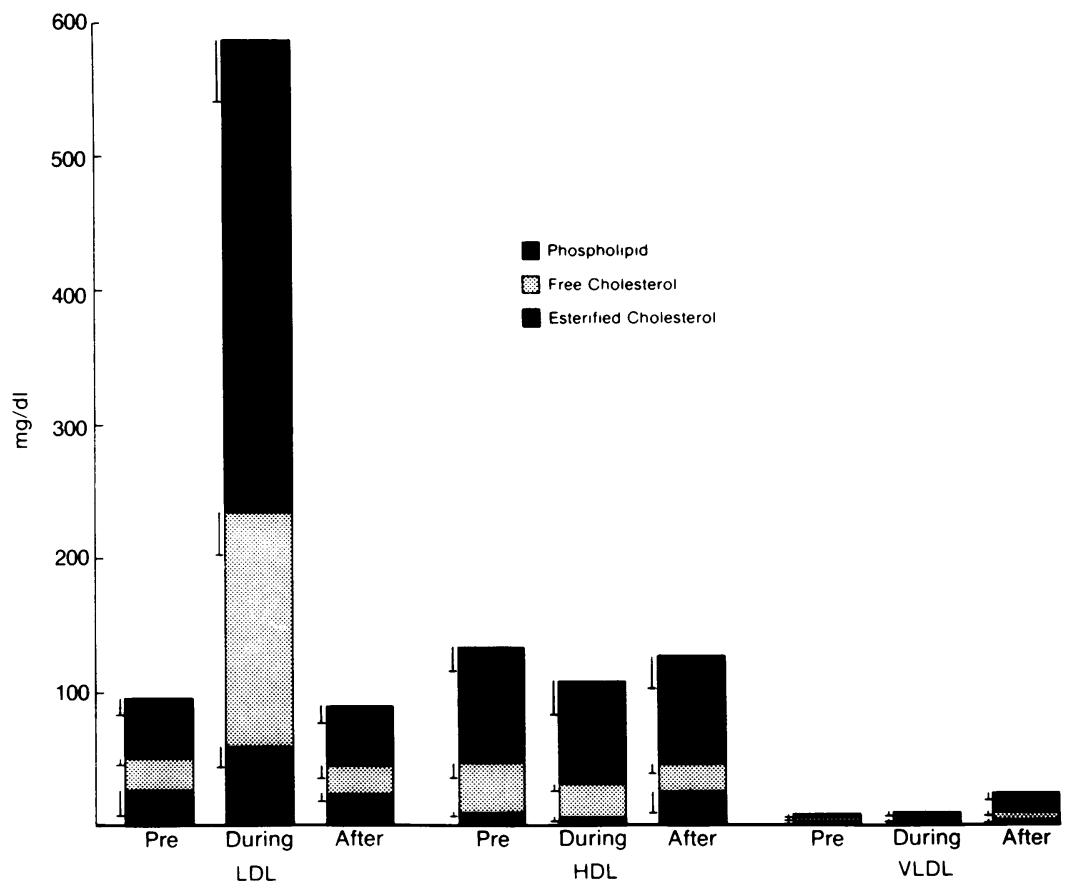

FIGURE 4 Composition of plasma lipoproteins $(n=5)$ before, during $(7-10 \mathrm{~d})$, and 6 wk after Intralipid infusion. The content of free cholesterol and phospholipid was significantly higher in LDL during Intralipid infusion $(P<0.01)$. The ratio of free cholesterol:esterified cholesterol was lower $(P<0.02)$ in HDL of neonates after infusion $(0.45 \pm 0.09)$ than during infusion $(3.48 \pm 0.51$; mean \pm SEM).

presence of Lp-X before $16 \mathrm{~h}$ could not be demonstrated by the electrophoretic procedure.

Because the major accumulation of free cholesterol and phospholipid was in LDL fraction, and because Lp-X was apparent by agar electrophoresis, an attempt was made to isolate and characterize $\mathrm{Lp}-\mathrm{X}$ using the technique of hydroxylapatite chromatography as described by Kostner et al. (14). After elution with $0.1 \mathrm{M}$

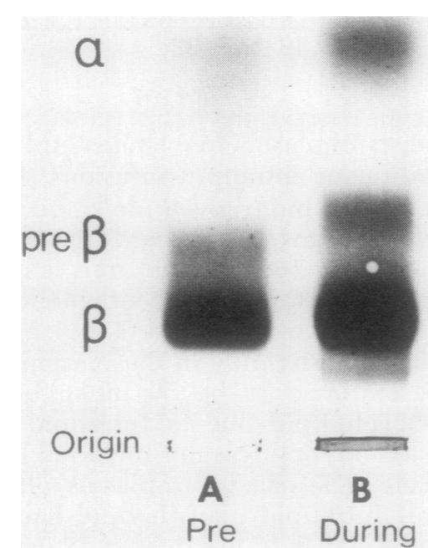

FIGURE 5 Agarose gel electrophoresis of plasma from a neonate. (A) Before and (B) at the end of $48 \mathrm{~h}$ of Intralipid infusion. phosphate buffer a lipoprotein was isolated with a composition essentially equivalent to $\mathrm{Lp}-\mathrm{X}$ isolated from a cholestatic patient (Table II). After $6 \mathrm{~d}$ of Intralipid infusion the free cholesterol of $\mathrm{Lp}-\mathrm{X}$ increased $30 \pm 10 \mathrm{mg} / \mathrm{dl}($ mean $\pm \mathrm{SD})$ and represented $\sim 25 \%$ of the total free cholesterol in LDL.

The small amount of protein (6\%) in Lp-X was entirely soluble in TMU, indicating the absence of apoB. No apoB was detected immunochemically, but albumin was detected after delipidation of the lipoprotein (data not shown). Further analysis by isoelectric focusing of the urea-soluble proteins of $\mathrm{Lp}-\mathrm{X}$ revealed (Fig. 7)

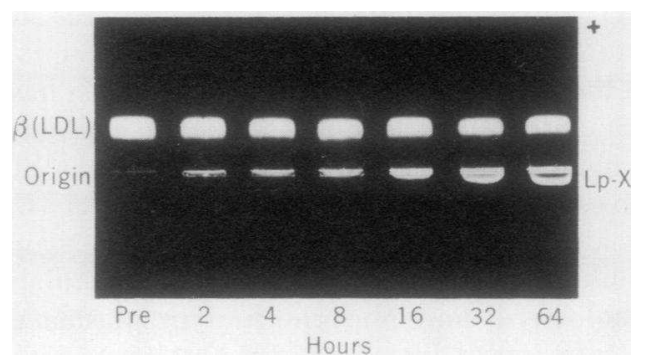

Figure 6 Agar gel electrophoresis of plasma of a neonate at various stages of Intralipid infusion ( $1 \mathrm{~g}$ Intralipid triglyceride/ $\mathrm{kg}$ body wt per $24 \mathrm{~h}$ ). The presence of cathodally migrating Lp$\mathrm{X}$ can be seen by $16 \mathrm{~h}$ of continous Intralipid infusion. 
TABLE II

Composition of Lipoproteins Isolated from $L D L(d=1.006-1.063)$ by

Hydroxylapatite Chromatography*

\begin{tabular}{|c|c|c|c|c|c|c|c|c|}
\hline \multirow[b]{3}{*}{$\begin{array}{c}\text { Eluate from } \\
\text { hydroxyapatite }\end{array}$} & \multirow[b]{3}{*}{ Subject! } & \multicolumn{7}{|c|}{ Component $\S$} \\
\hline & & \multirow[b]{2}{*}{ FC } & \multirow[b]{2}{*}{ CE } & \multirow[b]{2}{*}{ TG } & \multirow[b]{2}{*}{ PL } & \multicolumn{3}{|c|}{ Protein } \\
\hline & & & & & & Total & $\begin{array}{c}\text { TMU } \\
\text { soluble }\end{array}$ & $\begin{array}{c}\text { TMU } \\
\text { insoluble }\end{array}$ \\
\hline $0.1 \mathrm{M} \mathrm{PO}_{4}=$ & Cholestatic & 25.4 & 1.9 & 1.7 & 64.8 & 6.1 & 6.0 & 0 \\
\hline $0.1 \mathrm{MPO}_{4}=$ & Intralipid infused & 26.0 & 1.3 & 2.8 & 64.5 & 5.5 & 5.5 & 0 \\
\hline $0.65 \mathrm{M} \mathrm{PO}_{4}=$ & Intralipid infused & 15.1 & 20.5 & 5.4 & 38.5 & 20.4 & 4.5 & 15.9 \\
\hline $0.65 \mathrm{M} \mathrm{PO}_{4}=$ & Normal LDL & 6.3 & 42.5 & 5.1 & 22.5 & 23.5 & 1.1 & 22.3 \\
\hline
\end{tabular}

* LP-X was isolated in $0.1 \mathrm{M}$ phosphate buffer ( $\mathrm{pH}$ 6.8) from total LDL by hydroxylapatite chromatography. Residual LDL was obtained by a subsequent elution with 0.65 phosphate buffer. ‡ Cholestatic sample was obtained from an adult subject with obstructive jaundice and plasma Lp-X. To obtain sufficient material for analysis the LDL from $0.5 \mathrm{ml}$ of plasma, from three neonates infused with Intralipid (2-4 $\mathrm{g} / \mathrm{kg}$ body wt per d) for $6 \mathrm{~d}$, was first separated by hydroxylapatite chromatography. After the cholesterol was determined in the $0.1 \mathrm{M}$ and $0.65 \mathrm{M}$ phosphate buffer eluates, these fractions were pooled for complete lipid and protein analyses. The amount of total cholesterol in the $0.1 \mathrm{M} \mathrm{PO}_{4}=$ and $0.65 \mathrm{M} \mathrm{PO}_{4}=$ fractions was $30 \pm 10$ and $150 \pm 20 \mathrm{mg} / 100 \mathrm{ml}$, respectively. Recovery of lipoprotein cholesterol after hydroxylapatite chromatography was $90 \pm 5 \%$ $($ mean $\pm \mathrm{SD}$ ). The amount of TMU-insoluble protein in LDL increased from 35.2 to $39.6 \mathrm{mg} / 100 \mathrm{ml}$ in these subjects.

§ FC, free cholesterol; CE, cholesterol ester; TG, triglyceride; PL, phospholipid.

that a major protein focused at $\mathrm{pI} 6.0-6.2$, which is characteristic for human albumin under these conditions. A series of bands focusing between a pI of 5.5 and 5.9 were identical to the pI of isomorphs of apoE from normal human VLDL. ApoCII and apoCIII were also identified. When expressed as a percentage of total, optical density on the gel albumin accounted for $50 \%$, apoCII plus apoCIII for $30 \%$, and apoE for $10 \%$, with apoAI and unidentified peptides representing 5\%. Small amounts of apoE and apoAI have been identified in Lp-X isolated by zonal ultracentrifugation from cholestatic plasma (23).

The residual lipoproteins of the LDL fraction eluted with $0.65 \mathrm{M}$ phosphate buffer were abnormal (Table II). This fraction contained increased levels of free cholesterol, phospholipid, and TMU-soluble peptides, relative to normal $\mathrm{LDL}$; but apoB, identified by immunodiffusion against anti-LDL and quantitated by the amount of protein insoluble in TMU, was still a major component. In a single analysis of LDL pooled from three subjects there was a $10 \%$ increase in apoB during an infusion for $6 \mathrm{~d}$. Analyses of the urea-soluble proteins (Fig. 7) revealed that the TMU-soluble material was primarily composed of apoE $(60 \%)$ and apoCII plus apoCIII $(30 \%)$ with minimal amounts of albumin and unidentified peptides. Rechromatography on hydroxylapatite of the residual LDL fraction did not give Lp-X material that could be eluted with $0.1 \mathrm{M}$ phosphate buffer (data not shown). A similar particle has been observed in the LDL fraction of plasma from rats infused with Intralipid (24).
Initial studies on plasma specimens from infants who had been discontinued from Intralipid for 5-6 wk (Fig. 4) indicated that the plasma lipoprotein concentration and composition had returned to essentially preinfusion concentrations. To study shorter time intervals, plasma specimens were obtained at daily intervals after cessation of Intralipid infusion. Both plasma free cholesterol and phospholipid began to decrease within $24 \mathrm{~h}$ of termination of the infusion. By $96 \mathrm{~h}$ the free cholesterol levels had almost returned to preinfusion levels and plant sterols had disappeared, whereas plasma phospholipid levels were still somewhat elevated (Fig. 8). No Lp-X could be detected by agar gel electrophoresis $72 \mathrm{~h}$ after Intralipid discontinuation.

\section{DISCUSSION}

Parenteral lipid is an important adjunct in the management of the infant who cannot be fed orally and requires $100-150 \mathrm{kcal} / \mathrm{kg}$ per $\mathrm{d}$. Infants who are appropriate for gestational age and who have not lost excessive weight tolerate continuous Intralipid infusions, readily clearing $2-4 \mathrm{~g}$ of triglyceride/ $\mathrm{kg}$ body wt per $d(2-4)$ without developing hypertriglyceridemia. Despite a tolerance to the infused glyceride, hypercholesterolemia and hyperphospholipidemia develop as a function of the amount and duration of the Intralipid given, not only in infants, but also in adults (7-9) and experimental animals (24).

On the basis of the cholesterol content of the Intra- 


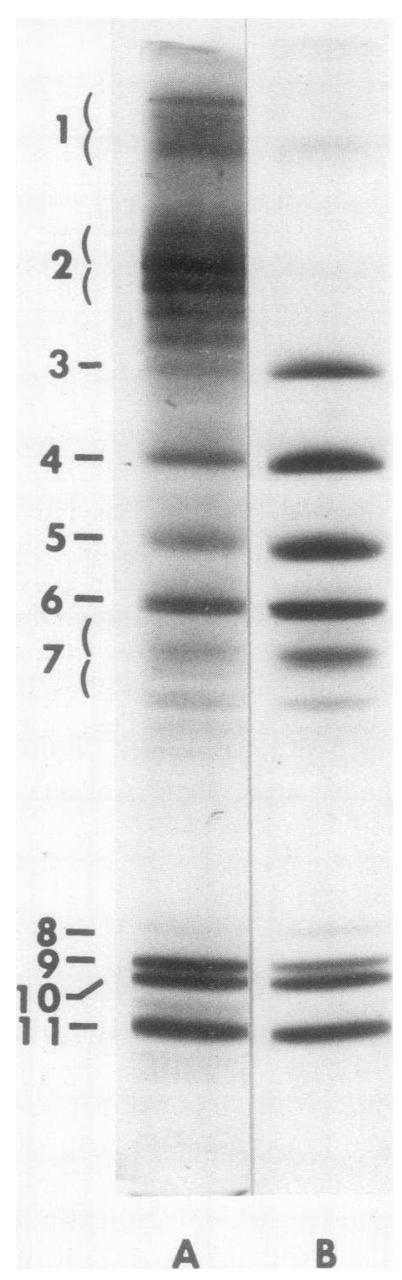

FIGURE 7 Isoelectric focusing of apoproteins in polyacrylamide gels $(10 \%)$ that contain $6 \mathrm{M}$ urea. Preparation of apoproteins and isoelectric focusing are described in Methods. Columns A and B represent Lp-X and residual LDL, respectively, obtained by hydroxylapatite chromatography of LDL from a neonate who received $2 \mathrm{~g}$ Intralipid triglyceride $/ \mathrm{kg}$ body wt per $24 \mathrm{~h}$ for $5 \mathrm{~d}$. Identification of apoproteins was based on isoelectric focusing of isolated apoprotein preparations from normal lipoproteins. 1, unidentified; 2, albumin; 3, corresponds to the unidentified band in column $\mathrm{B} ; 4, \operatorname{apoE}_{3} ; 5$, $\operatorname{apoE}_{2} ; 6$, apoE $E_{1} ; 7$, apoAI isomorphs; 8, apo CIIIo + apoAII; 9, apoCII; 10, apoCIII $; 11$, apoCIII 2 .

lipid and the amount infused, it was estimated that at least $50 \%$ of the free sterol that accumulated in plasma must have been derived from endogenous sources (Fig. 3). Similar calculations for the amount of phospholipid infused in Intralipid indicated that the bulk was cleared from plasma, but that the residual amount was sufficient to cause substantial increases in plasma phospholipids as a result of saturation of clearance pathways. The mechanism of phospholipid accumulation in plasma deserves comment because it may explain in part the hypercholesterolemia and the formation of $\mathrm{Lp}-\mathrm{X}$. In considering the mechanism of phospholipid clearance in the neonatal patients it is evident that the capacity was inadequate to remove the entire exogenous load at the infusion rates employed. Two enzymatic pathways involved in the removal of lecithin include $(a)$ lecithin-cholesterol acyltransferase (LCAT), an enzyme equally important in the deacylation of phospholipid as in the esterification of cholesterol and $(b)$ phospholipases that are heparin-releasable (25) and of hepatic origin (26). Although the quantitative importance of the phospholipases is unknown, the LCAT mechanism could theoretically be responsible for removal of $\sim 12-90 \%$ of the infused phospholipid. The plasma LCAT activity in man of $60-130 \mu \mathrm{mol}$ cholesterol esterified/liter of plasma per $h(27-29)$ is considerably less than the rate of infusion of phospholipid in our study $(145-580 \mu \mathrm{mol} /$ liter of plasma per h). In view of the fact that LCAT activity in newborns is reduced (30) it is reasonable to conclude that the LCAT mechanism was saturated with the substrate load, thus favoring accumulation of phospholipid in plasma.

It is important to note that $10 \%$ of the phospholipid in Intralipid is in mesophase and $90 \%$ of the phospholipid is associated with large glyceride-rich par-

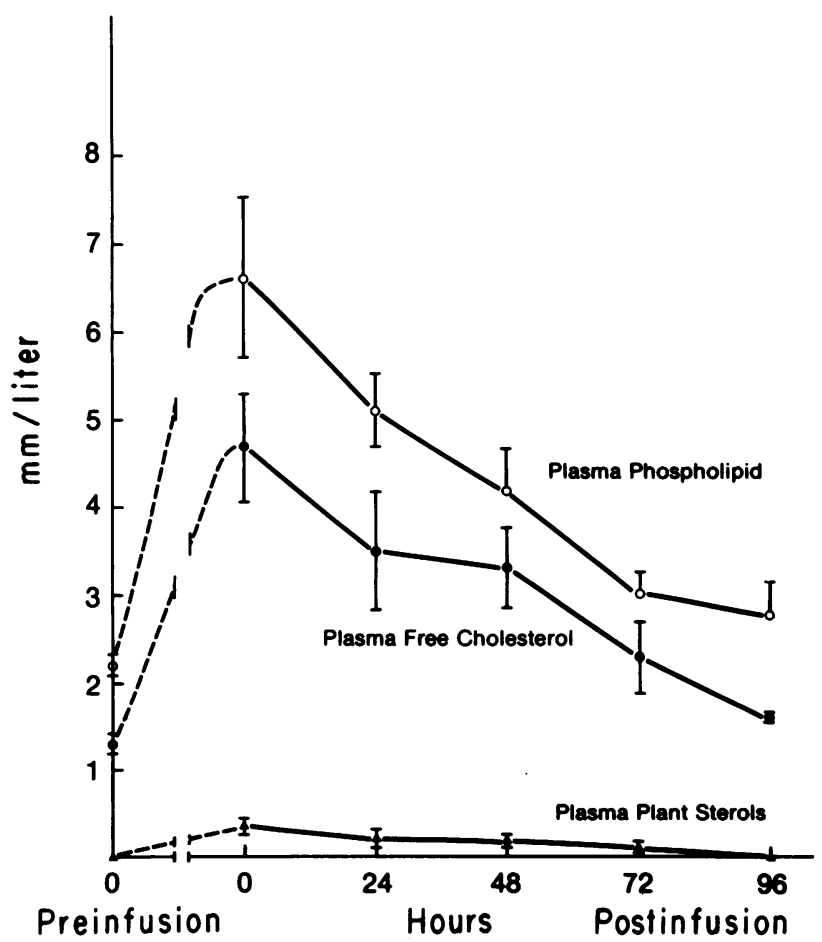

FIgURE 8 Return toward normal of plasma lipid levels after discontinuation of Intralipid infusions. Infants $(n=5)$ had been on Intralipid infusion (2-4 g triglyceride/ $\mathrm{kg}$ body wt) for 5-16 h. (O) Phospholipids; (O) free cholesterol; ( $\Delta$ ) plant sterols. 
ticles. The cholesterol:phospholipid ratio $(0.063)$ of both fractions is much lower than that found in Lp-X $(0.80)$. Because $>10 \%$ of the infused phospholipid was retained in plasma, both mesophase phospholipid and phospholipids remaining after the clearance of triglyceride-rich particles can be implicated in acquiring cholesterol from available tissue sources. This notion is supported by the observations: $(a)$ that the composition of phospholipids accumulating in plasma after Intralipid infusions is that of egg lecithin (31); (b) that the hypercholesterolemia after infusion of pure phospholipids in animals is a result of redistribution of cholesterol between tissue and plasma (32); and $(c)$ that phospholipid liposomes can leach cholesterol from erythrocyte membranes (33), and that phospholipid apoprotein complexes promote efflux of sterols from cells in tissue culture (34). A portion of the cholesterol accumulating in plasma may have been newly synthesized because phospholipid infusions are associated with enhanced hepatic cholesterogenesis $(35,36)$, presumably a result of increased human hydroxymethylglutaryl-coenzyme A reductase activity (37).

In our study, Lp-X was discerned after $16 \mathrm{~h}$ of Intralipid infusion when the increase in plasma phospholipid was 50-70\% above preinfusion levels (Fig. 6). Although Lp-X particles may be present but not detected at earlier times it is probable that the initial increment in phospholipid was accommodated by association with preexisting lipoproteins. This view is consistent with the data of Thompson et al. (8) who examined serum lipoproteins after a 4-h infusion of $50 \mathrm{~g}$ of Intralipid (containing $6.0 \mathrm{~g}$ of phospholipid) into healthy adults and observed no Lp-X formation. It is obvious that a much larger relative load of phospholipid must be administered and for longer periods of time before Lp-X appears; e.g., during TPN with Intralipid (6).

The exact origin of Lp-X is not established. Manzato et al. (38) suggested that in patients with cholestasis $\mathrm{Lp}$-X was derived from bile lipoproteins that contained phospholipid, cholesterol, and albumin, which refluxed into the blood stream and acquired C-apopeptides. In familial LCAT deficiency the accumulation of $\mathrm{Lp}-\mathrm{X}$ has been attributed to a failure to esterify cholesterol (39), which would be expected to lead to elevation of both free cholesterol and phospholipid levels. The extracellular formation of $\mathrm{Lp}-\mathrm{X}$ has been reported in perfused livers in rats with cholestasis (40). Although the exact process responsible for $\mathrm{Lp}-\mathrm{X}$ formation during Intralipid infusion cannot be discerned from this and previous (24) studies, an extracellular, physicochemical mechanism is proposed. As the level of plasma phospholipids exceeds the capacity for clearance, free cholesterol is mobilized by the lecithin-mesophase resulting in a stable phospholipid-cholesterol particle, which competes successfully with other lipoproteins for the apoproteins, and forming Lp-X. Alternatively, the apopeptides may be mobilized by the lecithin mesophase particles before their equilibration with free cholesterol. This hypothesis requires experimental confirmation.

It is significant that the rate of clearance of Lp-X after cessation of Intralipid infusion was similar to the clearance of Lp-X in cholestatic patients after operations to correct bile duct obstruction (41) or after plasma transfusion to patients with LCAT deficiency (42). In these instances a 50\% reduction in Lp-X occurred in $2-3 \mathrm{~d}$. In our study over $95 \%$ of the Lp-X disappeared in 2-4 d. These findings suggest that clearance of Lp-X is LCAT dependent because a reduction in Lp-X precursor (Intralipid mesophase) load or increasing enzymatic activities Lp-X levels are reduced. Furthermore the fact that $\mathrm{Lp}-\mathrm{X}$ occurred in conjunction with an absolute or relative deficiency in LCAT enzyme, whose function is entirely extracellular, supports the view that Lp-X occurs as an extracellular physicochemical event.

We have continued to use Intralipid (10\%) in the management of infants who cannot be fed in the normal manner. The absence of significant hemolysis and of renal disease clinically and at postmortem during short-term infusions, suggests that tissue changes were not associated with the presence of Lp-X. The effect of long-term infusions are yet to be established and deserve attention in view of the nephropathy associated with the Lp-X accumulation of LCAT deficiency.

\section{ACKNOWLEDGMENTS}

This work was supported by grants from the Medical Research Council of Canada and the Ontario Heart Foundation.

\section{REFERENCES}

1. Friedman, Z., A. Danon, M. T. Stahlamn, and J. A. Oates. 1976. Rapid onset of essential fatty acid deficiency in the newborn. Pediatrics 58: 640-649.

2. Gustafson, A., I. Kjellmer, R. Olegard, and L. Victorin. 1972. Nutrition in the low birthweight infants. I. Intravenous injection of fat emulsion. Acta Paediatr. Scand. 61: $149-158$.

3. Andrew, G., G. Chan, and D. Schiff. 1976. Lipid metabolism in the neonate. I. The effects of Intralipid infusion on plasma triglyceride and free fatty acid concentrations in the neonate. J. Pediatr. 88: 273-278.

4. Shennan, A. T., M. H. Bryan, and A. Angel. 1977. The effect of gestational age on Intralipid tolerance in newborn infants. J. Pediatr. 91: 134-137.

5. Franklin, F., J. B. Watkins, L. Heafitz, A. W. Clowes, and J. L. Breslow. 1976. Serum lipids during total parenteral nutrition with Intralipid (TPN-IL). Pediatr. Res. 10: 354 .

6. Griffin, E., A. Kuksis, C. Breckenridge, A. Jeejeebhoy, H. Bryan, and A. Angel. 1977. Hypercholesterolemia and lipoprotein-X (Lp-X) in total parenteral nutrition (TPN) with Intralipid. Clin. Res. 25: 311A.

7. Thompson, G. R., R. Segura, H. Hoff, and A. M. Gotto, Jr. 1975. Contrasting effects on plasma lipoproteins of 
intravenous versus oral administration of triglyceridephospholipid emulsion. Eur. J. Clin. Invest. 5: 373-384.

8. Thompson, G. R., A. Jadhav, M. Nava, and A. M. Gotto, Jr. 1976. Effects of intravenous phospholipid on low density lipoprotein turnover in man. Eur. J. Clin. Invest. 6: 241-248.

9. Jeejeebhoy, K. N., E. B. Marliss, G. H. Anderson, G. R. Greenberg, A. Kuksis, and C. Breckenridge. 1975. Lipid in parenteral nutrition: studies of clinical and metabolic features. In Symposium on Fat Emulsions in Parenteral Nutrition. American Medical Association, Chicago, Ill. 45-53.

10. Goldstein, A., L. Aronow, and S. M. Kalman. 1974. Rate of drug absorption. Principles of Drug Action: the basis of pharmacology. John Wiley \& Sons, Inc., New York. Second edition. 301-304.

11. Greenblat, D. J., and J. Kock-Weser. 1975. Clinical pharmacokinetics. N. Engl. J. Med. 293: 702-705.

12. Forget, P. P., J. Fernandes, and P. Haverkampbegman 1975. Utilization of fat emulsion during total parenteral nutrition in children. Acta Paediatr. Scand. 64: 377-384.

13. Hatch, F. T., and R. S. Lees. 1968. Practical methods for plasma lipoprotein analysis advances. J. Lipid Res. 6: $1-68$.

14. Kostner, G. M., W. Petek, and A. Holasek. 1974. Immunochemical measurement of lipoprotein X. Clin. Chem. 20: $676-681$.

15. Maguire, G. F., and W. C. Breckenridge. 1975. Agarose gel electrophoresis of plasma lipoproteins using the durrum cell. Clin. Biochem. 8: 161-168.

16. Seidel, D., H. Wieland, and C. Ruppert. 1973. Improved techniques for assessment of plasma lipoprotein patterns. I. Precipitation in gels after electrophoresis with polyanionic compounds. Clin. Chem. 19: 737-739.

17. Kane, J. P. 1973. A rapid electrophoretic technique for identification of subunit species of apoproteins in serum lipoproteins. Anal. Biochem. 53: 350-364.

18. Pagnan, A., R. J. Havel, J. P. Kane, and L. Kotite. 1977. Characterization of human very low density lipoproteins containing two electrophoretic population double pre $\beta$ lipoproteinemia and primary dysbeta lipoproteinemia. J. Lipid Res. 18: 613-622.

19. Reisner, A. H., P. Nemes, and C. Bucholtz. 1975. The use of Coomassie brilliant blue G-250 perchloric acid solution for staining in electrophoresis and isoelectric focusing on polyacrylamide gels. Anal. Biochem. 64: 509-516.

20. Lowry, O. H., N. J. Rosebrough, A. L. Farr, and R. J. Randall. 1951. Protein measurement with the Folin phenol reagent. J. Biol. Chem. 193: 265-275.

21. Kuksis, A., J. J. Myher, L. Marai, and K. Geher. 1975. Determination of plasma lipid profiles by automated gas chromatography and computerized data analysis. J. Chromatogr. Sci. 13: 423-430.

22. Seidel, D., P. Alaupovic, and R. H. Furman. 1969. A lipoprotein characterizing obstruction jaundice. Method for quantitative separation and identification of lipoproteins in jaundiced subjects. J. Clin. Invest. 48: 1211-1223.

23. Patsch, J. R., K. C. Aune, A. M. Gotto, Jr., and J. D. Morrisett. 1977. Isolation, chemical characterization and biophysical properties of three different abnormal lipoproteins: $\mathrm{Lp} \mathrm{X}_{1}, \mathrm{Lp} \mathrm{X}_{2}, \mathrm{Lp} \mathrm{X}_{3}$.J. Biol. Chem. 252: $2113-$ 2120.

24. Breckenridge, W. C., G. Kakis, and A. Kuksis. 1979. Identification of lipoprotein X-like particles in rat plasma following Intralipid infusion. Can.J. Biochem. 57: 72-82.

25. Vogel, W. C., and E. L. Bierman. 1967. Post heparin serum lecithinase in man and its positional specificity. J. Lipid Res. 8: 46-53.

26. Zieve, F. J., and L. Zieve. 1972. Post-heparin phospholipase and post heparin lipase have different tissue origins. Biochem. Biophys. Res. Commun. 47: 14801485.

27. Marcel, Y. L., and C. Vezina. 1973. A method for the determination of the initial rate of reaction of lecithin: cholesterol acyl transferase in human plasma. Biochim. Biophys. Acta. 306: 487-504.

28. Wallentin, L., and O. Vikrot. 1975. Lecithin: cholesterol acyl transfer in plasma of normal persons in relation to lipid and lipoprotein concentration. Scand. J. Clin. Lab. Invest. 35: 669-676.

29. Rose, H. G., and J. Juliano. 1976. Regulation of plasma lecithin cholesterol acyl transferase in man. I. Increased activity in primary hypertriglyceridemia. J. Lab. Clin. Med. 88: 29-43.

30. Cisternas, J. R., A. Llanos, R. Riquelme, and J. M. Caledon. 1976. Plasma lecithin: cholesterol acyltransferase activity in newborn term infants. J. Pediatr. 89: 323.

31. Kuksis, A., W. C. Breckenridge, J. J. Myher, and G. Kakis. 1978. Replacement of endogenous phospholipids in rat plasma lipoproteins during intravenous infusion of an artificial lipid emulsion. Can. J. Biochem. 56: 630-639.

32. Friedman, M., and S. O. Byers. 1957. Production of hypercholesterolemia in the rabbit by infusion of phosphatide or neutral fat. Proc. Soc. Exp. Biol. Med. 94: 452-456.

33. Cooper, R. A., E. C. Arner, J. S. Wiley, and S. J. Shattil. 1975. Modification of red cell membrane structure by cholesterol rich lipid dispersions. A model for the primary spur cell defect. J. Clin. Invest. 55: 115-126.

34. Stein, O., and Y. Stein. 1973. The removal of cholesterol from Landschuts Ascites cells by high density apolipoprotein. Biochim. Biophys. Acta. 326: 232-244.

35. Jakoi, L., and S. H. Quarfordt. 1974. The induction of hepatic cholesterol synthesis in the rat by lecithin mesophase infusions. J. Biol. Chem. 249: 5840-5844.

36. Byers, S. O., and M. Freidman. 1956. Independence of phosphatide induced hypercholesterolemia and hepatic function. Proc. Soc. Exp. Biol. Med. 92: 459-462.

37. Edwards, P. A. 1975. Effect of plasma lipoproteins and lecithin cholesterol dispersions on the activity of 3 hydroxy 3-methylglutaryl coenzyme A reductase of isolated rat hepatocytes. Biochim. Biophys. Acta. 409: 39-50.

38. Manzato, E., R. Fellin, G. Baggio, S. Walch, W. Neubeck, and D. Seidel. 1976. Formation of Lipoprotein X. Its relationship to bile compounds. J. Clin. Invest. 57: 12481260.

39. McConathy, W. J., P. Alaupovic, M. D. Curry, H. N. Magnani, H. Torsvik, K. Berg, and E. Gjone. 1973. Identification of lipoprotein families in familial lecithin: cholesterol acyltransferase deficiency. Biochim. Biophys. Acta. 326: 406-418.

40. Felker, T. E., R. L. Hamilton, and R. J. Havel. 1978. Secretion of lipoprotein-X by perfused livers of rats with cholestasis. Proc. Natl. Acad. Sci. U. S. A. 75: 3459-3463.

41. Ritland, S. 1975. Quantitative determination of the abnormal lipoprotein of cholestasis $\mathrm{Lp}-\mathrm{X}$ in liver disease. Scand. J. Gastroenterol. 10: 5-15.

42. Ritland, S., and E. Gjone. 1975. Quantitative studies of lipoprotein $\mathrm{X}$ in familial lecithin: cholesterol acyltransferase deficiency and during cholesterol esterification. Clin. Chim. Acta. 59: 109-119. 